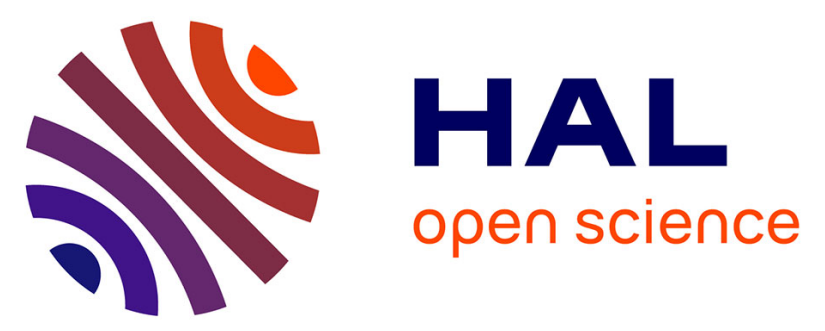

\title{
Efficient generation of data sets for one-shot statistical calibration of $\mathrm{RF} / \mathrm{mm}$-wave circuits
}

F. Cilici, G. Leger, Manuel J. Barragan, Salvador Mir, Estelle Lauga-Larroze, Sylvain Bourdel

\section{- To cite this version:}

F. Cilici, G. Leger, Manuel J. Barragan, Salvador Mir, Estelle Lauga-Larroze, et al.. Efficient generation of data sets for one-shot statistical calibration of $\mathrm{RF} / \mathrm{mm}$-wave circuits. International Conference on Synthesis, Modeling, Analysis and Simulation Methods and Applications to Circuit Design (SMACD 2019), Jul 2019, Lausanne, Switzerland. pp.17-20, 10.1109/SMACD.2019.8795238 . hal02166246

\section{HAL Id: hal-02166246 \\ https://hal.science/hal-02166246}

Submitted on 2 Oct 2020

HAL is a multi-disciplinary open access archive for the deposit and dissemination of scientific research documents, whether they are published or not. The documents may come from teaching and research institutions in France or abroad, or from public or private research centers.
L'archive ouverte pluridisciplinaire HAL, est destinée au dépôt et à la diffusion de documents scientifiques de niveau recherche, publiés ou non, émanant des établissements d'enseignement et de recherche français ou étrangers, des laboratoires publics ou privés.

\section{다(1) $\$$}

Distributed under a Creative Commons Attribution - NonCommerciall 4.0 International 


\title{
Efficient generation of data sets for one-shot statistical calibration of $\mathrm{RF} / \mathrm{mm}$-wave circuits
}

\author{
Florent Cilici*†, Gildas Leger ${ }^{\ddagger}$, Manuel J. Barragan*, Salvador Mir*, Estelle Lauga-Larroze ${ }^{\dagger}$, and Sylvain Bourdel ${ }^{\dagger}$ \\ ${ }^{*}$ Univ. Grenoble Alpes, CNRS, Grenoble INP ${ }^{1}$, TIMA F-38000 Grenoble, France \\ ${ }^{\dagger}$ Univ. Grenoble Alpes, Grenoble INP ${ }^{1}$, RFIC lab 38000 Grenoble, France \\ ${ }^{\ddagger}$ IMSE-CNM, Universidad de Sevilla-CSIC, 41092 Seville, Spain
}

\begin{abstract}
Millimeter-wave circuits in current nanometric technologies are especially sensitive to process variations, which can seriously degrade the device behavior and reduce fabrication yield. To tackle this issue, conservative designs and large design margins are widely used solutions. Another approach consists in introducing variable elements, also called tuning knobs, to allow post-fabrication tuning. One-shot statistical calibration techniques take advantage of advanced machine learning regression tools to propose a set of tuning knobs values that enhance the circuit performance based on simple measurements. Training the regression models require a huge amount of data covering the device performances, the effect of the tuning knobs and the simple measurements that guide the regression. In this work, we propose an efficient method for generating such a data set that reduces noticeably the size of the required training set for an accurate calibration.
\end{abstract}

\section{INTRODUCTION}

Continuous growth for fast systems and wireless communications has led the industry to millimeter-wave (mm-wave) frequencies. Current mm-wave integrated circuits are implemented in advanced nanometric technologies for which process variations are especially prominent. Excessive variations may significantly degrade the circuit performance, resulting in a reduction of the production yield. Thus, practical designs are devised with wide design margins, which usually incurs in large area and power overheads and lead to suboptimal trade-off solutions. A promising solution to this issue is to introduce calibration capabilities. This can be achieved by introducing variable elements, or tuning knobs, within the circuits to allow post-fabrication tuning. By controlling the tuning knobs, underperforming devices may be calibrated to reach better fabrication yields.

In the last few years, advanced one-shot calibration techniques based on machine learning algorithms have been proposed to avoid lengthy brute-force "test-and-tune" iterative calibration procedures. These techniques are used to find the best combination of tuning knob positions from a single set of simplified measurements. Calibration time in the production line is greatly reduced since the calibration is not iterative.

${ }^{1}$ Institute of Engineering Univ. Grenoble Alpes

This work has been partially funded by CNRS PICS project IndieTEST PICS07703 and the Spanish Government project TEC2015-68448-R (cofunded by FEDER program)
This procedure relies on statistical regression tools that are used to train models that capture the relationship between the measurements, the tuning-knob values and the performances of the Device Under Calibration (DUC).

The choice of low cost measurements and tuning-knobs are obviously key elements to take into account when devising a machine learning-based calibration strategy. However, there is a practical aspect that may limit the implementation of these approaches: building the machine learning regression models requires an important amount of data, including the low-cost measurements and DUC performances, varying tuning-knob values, for a significant number of different devices. The computational burden of generating these data in a simulation environment may be prohibitive, and this issue is accentuated when data comes from real measurements in the production line. In this paper we address this problem and propose a method to dramatically reduce the amount of data needed for building the regression models.

This paper is structured as follows. Section II reviews previous approaches for machine learning-based calibration of $\mathrm{RF} / \mathrm{mm}$-wave circuits. Section III details the proposed approach for the generation of training data while Section IV validates the method on a $60 \mathrm{GHz}$ Power Amplifier (PA) case study implemented in a $55 \mathrm{~nm}$ CMOS technology. Finally, Section V summarizes the main contributions of this work.

\section{PREVIOUS WORK}

In the last few years, different calibration strategies based on machine learning techniques have been proposed for RF and mm-wave circuits. One of the first machine learning calibration techniques was introduced in [1] where a $1.9 \mathrm{GHz}$ low noise amplifier (LNA) was calibrated by tuning two bias voltages. Calibration is based on a gradient search algorithm guided by a machine learning model that maps the output of an envelope detector to the circuit performances. Although it is a sound solution, the number of iterations to converge towards a good combination may become unfeasible for circuits with a large number of tuning knobs. This approach is further extended in [2] for the calibration of complete RF front-ends using the bias and supply voltages of mixers, LNA, and PA as tuning knobs. The search space in this case includes 7650 tuning knob combinations. 
One-shot techniques that avoid iterative optimizations were explored in [3], [4] to calibrate a $1.57 \mathrm{GHz}$ RF LNA. The performance of the fabricated LNA is estimated by using an embedded VCO and an output peak detector for exciting the LNA and acquiring its response, respectively. The bias voltages and power supply voltage are used as tuning knobs. The calibration strategy makes use of a machine learning model to predict the best values of the tuning knobs based on the output of the peak detector. This machine learning model is trained using a classic full factorial Design of Experiments (DOE) approach. Thus, over 27000 simulations were performed in [3] (3 tuning knobs, 3 knob positions, 1000 Monte Carlo instances), and over 49392 actual measurements were acquired in [4] (3 tuning knobs, 7 knob positions, 144 fabricated LNA samples) for data generation.

A similar approach was employed in [5], [6], where the one-shot calibration paradigm was applied to a $2.4 \mathrm{GHz} \mathrm{PA}$. The main difference lies in the use of non-intrusive dummy circuits that are sensitive to process variations. Four tuning knobs were proposed: 2 bias voltages and 2 supply voltages. Machine learning models map the output of the sensors to the optimum combination of tuning knobs. Data generation for training the calibration model is also based on a full factorial DOE combined with Monte Carlo simulation. The training data set in [5] is composed of 100 Monte Carlo circuit samples and 305 tuning knobs combinations, that is, a total of 30500 instances.

As previous works show, building calibration models based on DOE techniques is a time-consuming task that requires a huge number of simulations and/or measurements. The size of the required data set increases with the complexity of the DUC and the number of tuning knobs. In this paper we propose an efficient alternative to DOE for the generation of this data set.

\section{ONE-SHOT STATISTICAL CALIBRATION}

\section{A. Theoretical basis}

Let us consider a generic one-shot statistical calibration scenario for a DUC with $n$ specifications $\mathbf{P}=\left[P_{1}, P_{2}, \ldots, P_{n}\right]$ and $k$ tuning knobs $\mathbf{T K}=\left[T_{1}, T_{2}, \ldots, T_{k}\right]$. The main goal of statistical calibration techniques is to find a set of $l$ simple measurements (i.e., signatures) $\mathbf{S}=\left[S_{1}, S_{2}, \ldots, S_{l}\right]$ such that a regression function $f$ can be built as

$$
f:\left[T_{1}, \ldots, T_{k}, S_{1}, \ldots, S_{l}\right] \rightarrow\left[P_{1}, P_{2}, \ldots, P_{n}\right] .
$$

Once such a regression function has been determined, and by measuring vector $\mathbf{S}$ in a fabricated sample, function $f$ can be used for exploring the space of TK and maximize the performances $\mathbf{P}$. The regression function $f$ is obtained using a supervised machine learning algorithm. This procedure is divided into two stages: in a first stage -the training stagefor a population of circuits (i.e., the training set) both the performances and the signatures under different combinations of tuning knobs are extracted. A machine learning algorithm is then trained to regress the circuit performance from the signatures and tuning knob states. Then in a second stage the calibration stage - for circuits in the production line only the set of signatures is measured and the machine learning model previously obtained is used for determining the best combination of tuning knobs. The process has the advantage of avoiding iterative calibration loops, since the regression function allows finding the optimum calibration point in one shot.

\section{B. Data generation for training calibration models}

In order to build an accurate calibration model, the training set has to cover a significant variation range for both process variations and tuning knob positions. In fact, machine learning models do not extrapolate, so ideally the training data set should cover the full possible variation range. At the same time, the size of the training set has to be increased exponentially with the dimensionality of the problem in order to avoid sparse sets that would lead to inaccurate regressions.

The classical approach for generating the training data set relies on DOE techniques for defining an appropriate set of positions for the set of tuning knobs that covers the calibration space. Then, the effect of these tuning knob positions in the circuit performance is measured over a wide population of DUC samples to account for process variations. In these applications, the standard DOE approach is the so-called full factorial design, that would consider all possible combinations among a pre-defined set of positions for all the tuning knobs in the DUC. Thus, for a device with $k$ tuning knobs, if we consider that each tuning knob has $p_{i}$ pre-defined positions, where $i=1, \ldots, k$, then a total of $M=\prod_{i=1}^{k} p_{i}$ combinations have to be considered for each circuit sample. In a simulation environment, these circuit samples are generated by Monte Carlo sampling and, ideally, should cover the full process variation range of the DUC.

Fig. 1a shows a visual representation of the training data set obtained by DOE for a generic one-shot statistical calibration scenario. The data set contains the set of performances and signatures for all tuning knob combinations, in the considered Monte Carlo process variation range.

Standard DOE techniques are feasible for a reduced number of knobs and knob positions. For example, an analog tuning knob may be usually represented with only three values: min, max, and typ. However, accurately capturing non-linear knob behavior and complex knob interactions require a higher granularity. In this paper, we propose an alternative to DOE techniques for statistical calibration that allows to capture accurately the behavior of the tuning knobs and their interactions, while reducing the size of the training data set. Instead of sampling a limited set of pre-defined values for the tuning knob positions, we propose to randomly sample each knob position. In this way, in a simulation environment, both process and tuning knob variations may be similarly generated within a Monte Carlo simulation, as visually represented in Fig. 1b. A simple implementation of this technique consists in associating a probability density function to each tuning knob position that covers its full variation range. A uniform probability density function would produce a uniform variation of the tuning knobs for the samples in the training data set. Depending on 


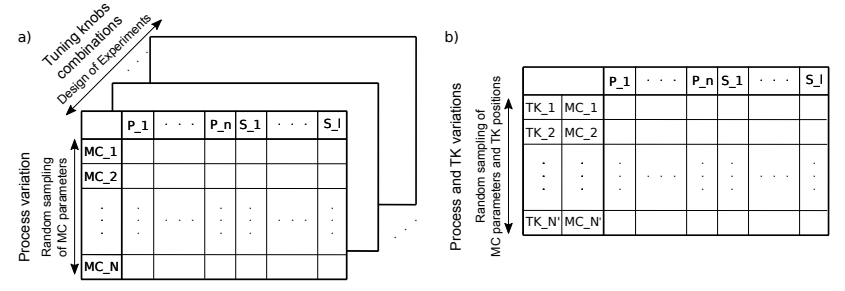

Fig. 1. Generation of training data sets using a) Full factorial DOE and b) random sampling.

the application scenario, the probability density function can be also tailored to each particular tuning knob to enrich the information in the data set. In this paper we demonstrate that this random sampling strategy reduces significantly the size of the training data set necessary for capturing process and tuning knob variations.

\section{CASE STUdY}

\section{A. Two-stage $60 \mathrm{GHz}$ power amplifier with tuning capabilities}

A two-stage $60 \mathrm{GHz}$ power amplifier has been chosen as a case study. It has been designed in a $55 \mathrm{~nm}$ CMOS technology from STMicroelectronics. Its electrical schematic is presented in Figure 2 and its main performance figures are summarized in Table I. Six tuning-knobs have been devised: two gate bias voltages $\left(V_{B 1}\right.$ and $\left.V_{B 2}\right)$ and four programable decoupling cells used as termination loads for the stubs $\left(C_{V 1}, C_{V 2}, C_{V 3}\right.$ and $C_{V 4}$ ), controlled by voltages $V_{C 1}, V_{C 2}, V_{C 3}$ and $V_{C 4}$. The design of the case study is detailed in [7]. The statistical calibration procedure relies on a set of signatures provided by non-intrusive process variation monitors. These sensors have been designed following the methodology presented in [8] to accurately capture process variations.

TABLE I

PERFORMANCES AND DESIGN PARAMETERS OF THE POWER AMPLIFIER

\begin{tabular}{lc}
\hline DC current, $I_{D C}$ & $51.1 \mathrm{~mA}$ \\
Power supply, $V_{D D}$ & $1.2 \mathrm{~V}$ \\
Gain $\left(S_{21}\right)$ & $9.3 \mathrm{~dB} @ 60 \mathrm{GHz}$ \\
Power Added Efficiency, PAE & $12.9 \%$ \\
Output referred 1dB-Compression Point, $C P_{1 d B}$ & $7.1 \mathrm{dBm}$ \\
Saturation output power, $P_{\text {sat }}$ & $11.6 \mathrm{dBm}$ \\
$S_{11}$ & $<-20 \mathrm{~dB}$ \\
$S_{12}$ & $<-30 \mathrm{~dB}$ \\
\hline
\end{tabular}

\section{B. One-shot statistical calibration results}

A one-shot statistical calibration strategy was used for mapping the output of the built-in process monitors to the optimum position of each tuning knob in order to compensate performance degradations due to process variations. As discussed before, a key point for generating the regression function is the definition of an appropriate training data set. In this section, we show a direct comparison between the classical DOE sampling and the proposed random sampling of knob positions.

The presented calibration results were obtained through post-layout Monte Carlo simulations using the spectreRF simulator. Four different calibration scenarios were considered for comparison purposes. The training data sets in each scenario contain:

a) 5000 instances of the DUC: process variations and tuningknobs values are generated using the proposed random sampling strategy. Uniform probability density functions were associated to each tuning knob covering its full variation range.

b) 5040 instances of the DUC: 35 Monte Carlo iterations were performed for a set of $2^{4} \times 3^{2}=144$ selected knobs combinations (i.e., $\min$ and $\max$ for each varactor and $\min$, typ and max for the two gate bias voltages).

c) 50400 instances of the DUC: 350 Monte Carlo iterations were performed for a set of 144 selected knobs combinations (same as in scenario b)).

d) 50301 instances of the DUC: 69 Monte Carlo iterations were performed for a set of $3^{6}=729$ selected knobs combinations (i.e., $\min$, typ and $\max$ for the 6 tuning knobs).

Data sets a) and b) allows a direct comparison between the proposed data generation methodology and the DOE technique used in previous work [3]-[6] using a similar number of training samples. Data sets c) and d) rely as well on DOE, but we have multiplied by 10 the number of training instances of the PA compared to b). Data set c) should offer a better coverage of process variations, since the number of Monte Carlo iterations have been increased, while data set d) should improve the coverage of the tuning knob impact on the PA performance.

Following the statistical one-shot calibration methodology described in section III, we have trained regression functions $f$ for each performance of the PA, for the four training data sets. This regression function is used for calibrating an independent set of 500 PA instances generated by Monte Carlo simulation. The obtained results are compared based on a figure of merit (FOM) defined as,

$$
F O M=\frac{\sigma_{\text {performance }}}{\mu_{\text {error }}},
$$

where $\sigma_{\text {performance }}$ is the standard deviation of the performance and $\mu_{\text {error }}$ is the RMS prediction error.

Table II displays the FOM results for the four considered scenarios. For scenarios a) and b) (i.e, 5000 samples) and for all the performances, the obtained FOMs are significantly better using random sampling than with the DOE technique. For scenarios c) and d), even with 10 times more data in the training set, results provided by the DOE method still do not match scenario a) that uses random sampling. Regarding scenarios c) and d), it appears that data set c) lacks information about the effect of the tuning knobs for large signal performances and that data set d) lacks information about process variations, especially for small-signal performances.

In order to show a direct comparison of the regression capabilities of the trained regression function $f$, Fig. 3 represents the scatterplot of the predicted versus actual values of the PA small signal gain for the 500 instances in the independent 


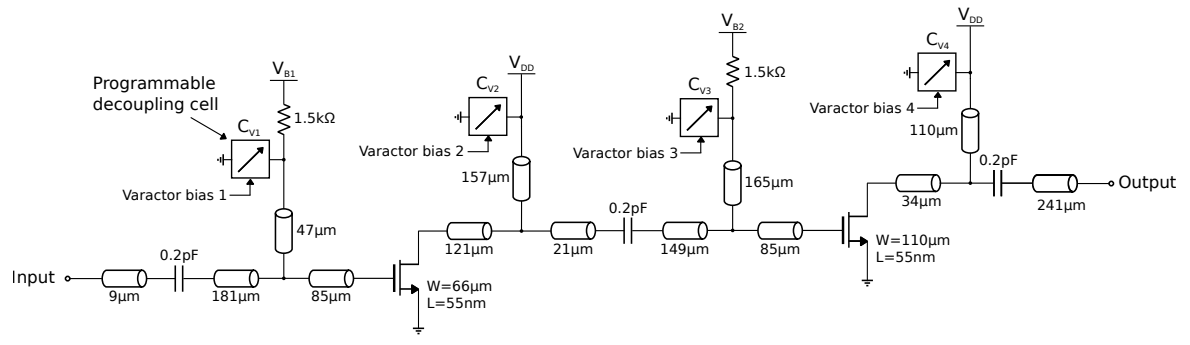

Fig. 2. Transistor-level schematic of the power amplifier under calibration.

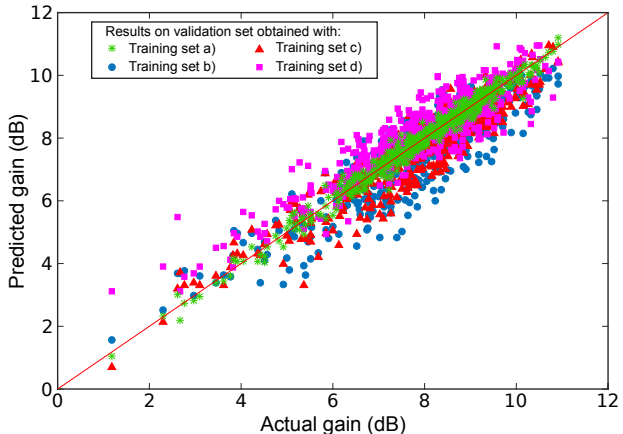

Fig. 3. Scatterplot of predicted versus actual PA gain for the samples in the validation set.

TABLE II

COMPARISON OF PA PERFORMANCES FIGURES OF MERIT FOR DIFFERENT TRAINING SETS

\begin{tabular}{|c|cccc|}
\cline { 2 - 5 } \multicolumn{1}{c|}{ Training set } & \multicolumn{4}{c|}{ Figure of Merit } \\
\hline Performance & a) & b) & c) & d) \\
\hline$I_{D C}$ & 90 & 59 & 68.5 & 68.3 \\
$C P_{1 d B}$ & 8.3 & 2.8 & 3.8 & 5.45 \\
$P A E$ & 7.2 & 2.7 & 3.45 & 4.4 \\
$P_{\text {sat }}$ & 6.4 & 2.0 & 3.4 & 4.0 \\
$S_{21}$ & 6.4 & 2.0 & 2.6 & 2.3 \\
$S_{11}$ & 4.6 & 1.2 & 2.5 & 2.45 \\
\hline
\end{tabular}

validation set. It is clear that training the regression model with data set a) (in green) results in a better accuracy.

For further insight, Fig. 4 shows the FOM corresponding to the PAE calibration for scenarios a), c) and d) as a function of the number of samples in the training set (obtained by random sub-sampling of the data sets). It is interesting to notice the linear behavior of the FOM calculated on set a) as the size increases. In comparison, the FOM calculated on set c) tends to saturate as the number of training instances increases, probably due to the lack of information about the effect of the tuning knobs. Similarly, data set d) shows the same behavior, but this time probably due to a lack of process variation information. It seems clear that the proposed random sampling methodology offers a better coverage of the training space while reducing the number of required simulations.

\section{Conclusions}

This work presented an efficient methodology for the generation of appropriate training data sets for statistical calibration applications. The proposed technique is based on the joint

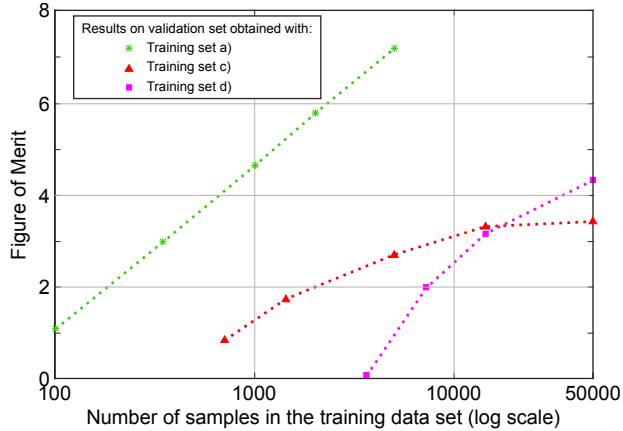

Fig. 4. Figure of merit for the prediction of the PAE as a function of the size of the training data set.

random sampling of both process and tuning knob position variations. Compared to previous works based on DOE, this method dramatically reduces the size of the required training data set for an accurate training of statistical calibration models. Obtained results on a tunable 2 -stage $60 \mathrm{GHz}$ case study in a $55 \mathrm{~nm}$ technology demonstrate the feasibility of the approach.

\section{REFERENCES}

[1] V. Natarajan, R. Senguttuvan, S. Sen, and A. Chatterjee, "ACT: Adaptive Calibration Test for Performance Enhancement and Increased Testability of Wireless RF Front-Ends," in 26th IEEE VLSI Test Symposium (VTS), April 2008, pp. 215-220.

[2] V. Natarajan, A. Banerjee, S. Sen, S. Devarakond, and A. Chatterjee, "Yield Recovery of RF Transceiver Systems Using Iterative TuningDriven Power-Conscious Performance Optimization," IEEE Design \& Test, vol. 32, no. 1, pp. 61-69, Feb 2015.

[3] N. Kupp, H. Huang, P. Drineas, and Y. Makris, "Post-production performance calibration in analog/RF devices," in 2010 IEEE International Test Conference, November 2010, pp. 1-10.

[4] Y. Lu, K. Subramani, H. Huang, N. Kupp, and Y. Makris, "Silicon Demonstration of Statistical Post-Production Tuning," in IEEE Computer Society Annual Symposium on VLSI, July 2015, pp. 628-633.

[5] M. Andraud, H. Stratigopoulos, and E. Simeu, "One-shot calibration of $\mathrm{RF}$ circuits based on non-intrusive sensors," in 51st ACM/EDAC/IEEE Design Automation Conference (DAC), June 2014, pp. 1-6.

[6] _ - "One-Shot Non-Intrusive Calibration Against Process Variations for Analog/RF Circuits," IEEE Transactions on Circuits and Systems I: Regular Papers, vol. 63, no. 11, pp. 2022-2035, Nov 2016.

[7] F. Cilici, M. J. Barragan, S. Mir, E. Lauga-Larroze, S. Bourdel, and G. Leger, "Yield Recovery of mm-Wave Power Amplifiers using Variable Decoupling Cells and One-Shot Statistical Calibration," in IEEE International Symposium on Circuits and Systems (ISCAS), May 2019. pp. 1-5.

[8] F. Cilici, M. J. Barragan, S. Mir, E. Lauga-Larroze, and S. Bourdel, "Assisted test design for non-intrusive machine learning indirect test of millimeter-wave circuits," in IEEE 23rd European Test Symposium (ETS), May 2018, pp. 1-6. 